\title{
Central Serous Chorioretinopathy With Multiple Leakage Points in a Young Female Patient Without Detected Risk Factors
}

\author{
Irini P. Chatzirallia, b, Evgenia Kanonidou ${ }^{\mathrm{a}}$, Christina Kanonidou, \\ Alexandros Chatzirallis ${ }^{\mathrm{a}}$, Leonidas Papazisis ${ }^{\mathrm{a}}$
}

\begin{abstract}
Central Serous Chorioretinopathy (CSR) is characterized by the accumulation of subretinal fluid at the posterior pole of the fundus, causing serous detachment of the neurosensory retina and/or retinal pigment epithelium. CSR typically affects young and middle-aged men between 20 and 40 years old with type-A personality and is associated with emotional stress. A 21-year-old non-pregnant female presented with a 2-day history of acute, sudden, painless loss of vision and metamorphopsia at the right eye. She complainted also for a central scotoma. On examination of the right eye, the best-corrected visual acuity (BCVA) was 3/10. Dilated fundus examination revealed a neurosensory serous detachment and the diagnosis of CSR was confirmed by fluorescein angiography and optical coherence tomography (OCT). Interestingly, the fluorescein angiography revealed multiple leakage points of the dye related to retinal pigmented epithelium defects, while OCT demonstrated a significantly increased retinal thickness. The most astonishing feature of this case report is that it concerns a female patient with acute CSR without any detected risk factors that was characterized by significantly high retinal thickness in OCT in parallel with multiple leakages in fluorescein angiography. As a result, CSR should be taken into account in the differential diagnosis of sudden, painless loss of vision, although the patient may be female without risk factors.
\end{abstract}

Keywords: Central serous retinopathy; Risk factors; OCT; FA

\section{Introduction}

Central Serous Chorioretinopathy (CSR) is characterized by

Manuscript accepted for publication February 23, 2012

${ }^{\mathrm{a}}$ Department of Ophthalmology, Veroia General Hospital, Veroia, Greece

${ }^{\mathrm{b}}$ Corresponding author: Irini P. Chatziralli, 28, Papanastasiou street, Athens 17342, Greece. Email: eirchat@yahoo.gr

doi: $10.4021 / \mathrm{jmc} 580 \mathrm{w}$ the accumulation of subretinal fluid at the posterior pole of the fundus, causing serous detachment of the neurosensory retina and/or retinal pigment epithelium $[1,2]$. CSR typically affects young and middle-aged adults (between ages 20 and 40 years) with men being affected more commonly than women by approximately 10 to one [1-5]. It has been associated with type-A personality, elevated endogenous cortisol and corticosteroid therapy in various forms $[1,5$, 6]. The main symptoms range from acute loss of vision to metamorphopsia and scotomas [1-6]. We report the case of a young female patient who developed acute CSR without recognisable risk factors.

\section{Case Report}

A 21-year-old non-pregnant female presented with a 2-day history of acute, sudden, painless loss of vision and metamorphopsia at the right eye. She was also complaining for a central scotoma. Her past medical, as well as her ocular history, were clear. On examination, best-corrected visual acuity (BCVA) was $3 / 10$ in the right eye and $10 / 10$ in the left eye. Metamorphopsia was confirmed using the Amsler grid. Dilated fundus examination revealed a localized round serous detachment at the macula and she referred to undergo fluorescein angiography (FA) and optical coherence tomography (OCT). Fluorescein angiography demonstrated multiple leakage points of the dye related to retinal pigmented epithelium defects (Fig.1). Optical coherence tomography showed full thickness detachment of the neurosensory retina with dense subretinal deposits and a significantly increased central retinal thickness of $603 \mu \mathrm{m}$ at the macula, confirming the diagnosis of CSR (Fig. 2). The left eye had no pathological findings and normal OCT.

\section{Discussion}

Central Serous Retinopathy typically affects young and middle-aged men between 20 and 40 years old with type-A personality and is associated with emotional stress [5]. It has also been described in women, usually in pregnancy [7]. Our 


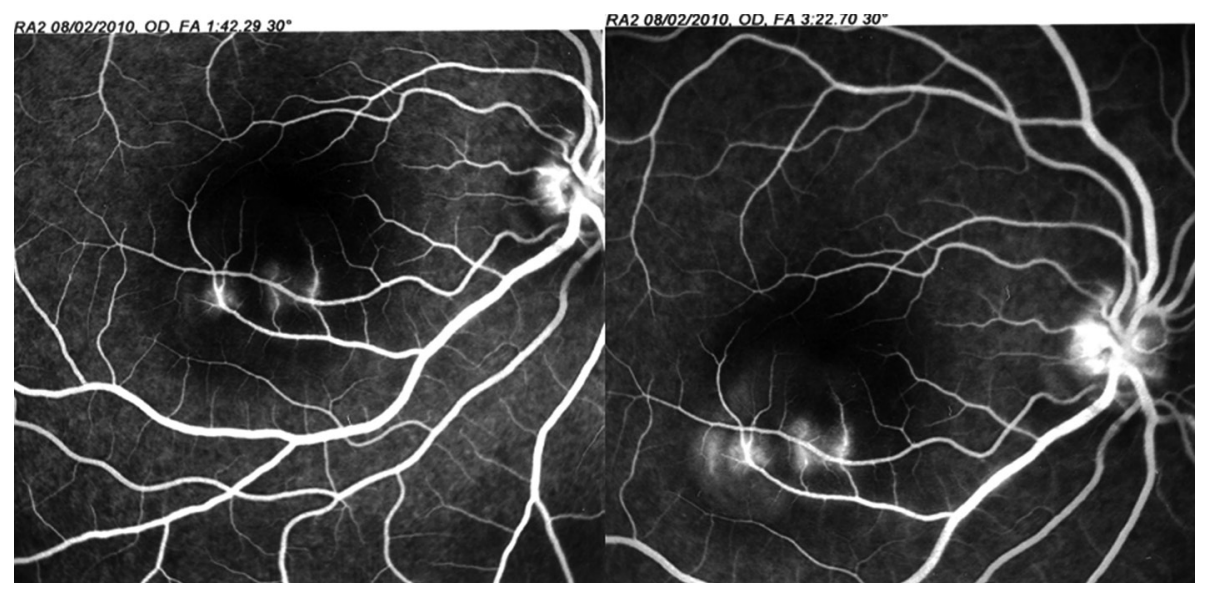

Figure 1. Fluorescein angiography findings of our patient: Multiple leakage points of the dye were observed during the late phase.

patient was a non-pregnant female without any professional or emotional stress and she could not be considered as typeA personality.

Central Serous Retinopathy is associated with elevated serum cortisol levels, endogenous hypercortisolism, use of costicosteroids in various forms and high testosterone levels, specially related to corticosteroid or testosterone therapy [1, $3,4,7]$. Our patient was not under any corticosteroid nor testosterone therapy and in biochemical examination, the hormones serum level (FSH, LH, PRL, PRG, E2, Testosterone, DHEA-S, $\beta$-hcg) was normal, apart from cortisol which was a little bit elevated $(21.1 \mu \mathrm{g} / \mathrm{dl}$, normal ranges: $6.2-19.4 \mu \mathrm{g} /$ dl).

Other risk factors for the development of CSR are $H$. pylori [8], hypertension, the use of phychopharmacological agents $[9,10]$, alcohol use [10], antibiotic use [10], allergic respiratory disease [10], anthrax vaccination [11] and systemic lupus erythematosus or other collagen disease [2]. Our patient had no history of gastritis, stomach or duodenum ulcer and she was never diagnosed for H. pylori. She had also neither hypertension nor any systematic disease and she took no medicaments at all.

The main symptoms of CSR are acute, painless loss of vision, a relative central scotoma, metamorphopsia, micropsia and dyschromatopsia [2-4]. Our patient had all these symptoms, apart from dyschromatopsia. Specifically, she complainted about a dark spot in the centre of her visual field in the right eye and metamorphopsia.

The diagnosis of CSR is based on the ophthalmologic findings and results of FA and OCT. The prominent angiographic characteristic is that of an expanding point of fluorescein leakage under serous detachment of the sensory retina, without signs of subretinal neovascularizarion. Usually, one or two leakage points are seen [2]. In our case, there are multiple leakage points of the dye, which is rare. Moreover, OCT measures the retinal thickness and the maximal retinal thickness at the macula in similar cases is usually $381 \pm 82$ $\mu \mathrm{m}$ [12-14]. In our case, the central subfield thickness was $603 \mu \mathrm{m}$.

The patient was treated with acetazolamide, as the effect of acetazolamide in the management of chronic macular edema and of serous retinal detachment is well established [14].

The most astonishing feature of this case report is that it concerns a young female patient with acute CSR without any detected risk factors that was characterized by significantly increased retinal thickness in OCT in parallel with multiple

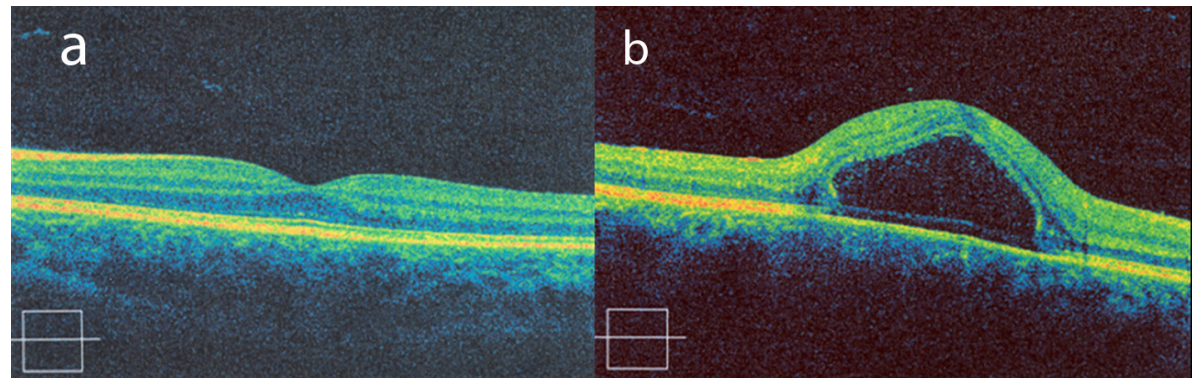

Figure 2. OCT findings of our patient: (a) normal OCT of the left eye, (b) full thickness detachment of the neurosensory retina at the macula of the right eye. 
leakages of the dye in fluorescein angiography. As a result, CSR should be taken into account in the differential diagnosis of sudden, painless loss of vision, although the patient may be female without risk factors.

\section{Conflict of Interest and Funding Sources}

The authors declare no conflict of interest.

\section{References}

1. Bouzas EA, Scott MH, Mastorakos G, Chrousos GP, Kaiser-Kupfer MI. Central serous chorioretinopathy in endogenous hypercortisolism. Arch Ophthalmol. 1993;111(9):1229-1233.

2. Wang M, Munch IC, Hasler PW, Prunte C, Larsen M. Central serous chorioretinopathy. Acta Ophthalmol. 2008;86(2):126-145.

3. Zakir SM, Shukla M, Simi ZU, Ahmad J, Sajid M. Serum cortisol and testosterone levels in idiopathic central serous chorioretinopathy. Indian J Ophthalmol. 2009;57(6):419-422.

4. Bouzas EA, Karadimas P, Pournaras CJ. Central serous chorioretinopathy and glucocorticoids. Surv Ophthalmol. 2002;47(5):431-448.

5. Yannuzzi LA. Type A behavior and central serous chorioretinopathy. Trans Am Ophthalmol Soc. 1986;84:799845.

6. Al-Mujaini A, Wali U, Ganesh A, Montana C. Natural course of central serous chorioretinopathy without subretinal exudates in normal pregnancy. Can J Ophthalmol. 2008;43(5):588-590.

7. Ahad MA, Chua CN, Evans NM. Central serous chorioretinopathy associated with testosterone therapy. Eye (Lond). 2006;20(4):503-505.

8. Misiuk-Hojlo M, Michalowska M, Turno-Krecicka A. Helicobacter pylori--a risk factor for the developement of the central serous chorioretinopathy. Klin Oczna. 2009;111(1-3):30-32.

9. Tittl MK, Spaide RF, Wong D, Pilotto E, Yannuzzi LA, Fisher YL, Freund B, et al. Systemic findings associated with central serous chorioretinopathy. Am J Ophthalmol. 1999;128(1):63-68.

10. Haimovici R, Koh S, Gagnon DR, Lehrfeld T, Wellik S, Central Serous Chorioretinopathy Case-Control Study G. Risk factors for central serous chorioretinopathy: a case-control study. Ophthalmology. 2004;111(2):244249.

11. Foster BS, Agahigian DD. Central serous chorioretinopathy associated with anthrax vaccination. Retina. 2004;24(4):624-625.

12. Montero JA, Ruiz-Moreno JM. Optical coherence tomography characterisation of idiopathic central serous chorioretinopathy. Br J Ophthalmol. 2005;89(5):562-564.

13. Springer C, Volcker HE, Rohrschneider K. [Central serous chorioretinopathy--retinal function and morphology: microperimetry and optical coherence tomography]. Ophthalmologe. 2006;103(9):791-797.

14. Gonzalez C. [Serous retinal detachment. Value of acetazolamide]. J Fr Ophtalmol. 1992;15(10):529-536. 